\title{
émulations
}

\section{Analyser et qualifier la persuasivité des discours contenus dans un jeu vidéo}

\section{Le cas de la récession de Possum Springs}

\author{
Esteban Giner
}

Émulations - Revue de sciences sociales

2019, n³0, «Comment les jeux font-ils société. Contenus, pratiques et médiations ludiques ».

\section{Article disponible à l'adresse suivante}

https://ojs.uclouvain.be/index.php/emulations/article/view/giner

\section{Pour citer cet article}

Esteban Giner, «Analyser et qualifier la persuasivité des discours contenus dans un jeu vidéo. Le cas de la récession de Possum Springs », Émulations, n³0, Mise en ligne le 3 septembre 2019.

DOI : 10.14428/emulations.030.07

Distribution électronique : Université catholique de Louvain (Belgique) : ojs.uclouvain.be

(C) Cet article est mis à disposition selon les termes de la Licence Creative Commons Attribution, Pas d'Utilisation Commerciale 4.0 International. http://creativecommons.org/licenses/by-nc/4.0/

Éditeur : Émulations - Revue de sciences sociales / Presses universitaires de Louvain https://ojs.uclouvain.be/index.php/emulations

ISSN électronique : 1784-5734

PUL PRESSES

UNIVERSITAIRES

DE LOUVAIN 


\title{
Analyser et qualifier la persuasivité des discours contenus dans un jeu vidéo
}

\author{
Le cas de la récession de Possum Springs
}

Esteban Giner $^{1}$

\begin{abstract}
[Résumé] Afin de dépasser les concepts d'expressive games et de persuasive games, nous proposons un cadre sémio-pragmatique en mobilisant des outils des sciences de l'éducation et ce, afin de qualifier la persuasivité, le caractère persuasif, d'un game design. Nous concluons à la nécessité d'envisager la persuasivité d'un jeu vidéo sous la forme d'un continuum. Les discours sont alors le produit de controverses entre la machine et l'opérateur-trice et ce au travers de situations de communication plus ou moins persuasives, plus ou moins expressives. Afin de développer notre réflexion, nous mobilisons le jeu Night In The Woods qui propose à son joueur ou sa joueuse de parcourir Possum Springs, une ville fictive de la Rust Belt dont la désindustrialisation fut aggravée par la crise des subprimes. Porteur de nombreux discours sur la situation des ménages après la crise, le jeu se révèle expressif sur cette problématique bien qu'il puisse être persuasif sur les autres thématiques qu'il aborde.
\end{abstract}

Mots-clés : Crise des subprimes, étude des discours, game design, persuasivité, continuum persuasif, expressive games

[Abstract] In order to go beyond the concepts of expressive games and persuasive games, we propose a semio-pragmatic framework by mobilizing educational sciences tools to qualify the persuasiveness of a game design. We conclude that we need to consider the persuasiveness of a video game in the form of a continuum. The discourses are then the product of controversies between the machine and the operator through situations of communication more or less persuasive, more or less expressive. In order to develop our thinking, we mobilize the game Night In The Woods which proposes to its player to browse Possum Springs, a fictional city of the Rust Belt whose deindustrialization was aggravated by the subprime crisis. Bearer of many discourses about households after the crisis, the game is expressive on this issue even though it can be persuasive on other topics it addresses.

Keywords: Subprime crisis, discourse analysis, game design, persuasiveness, persuasive continuum, expressive games

«Tout s'effondre. Possum Springs saigne à en mourir. Et bientôt nous serons tous morts et cette ville ne sera plus que champs et forêts. »² (le leader du Culte de Possum Springs)

\footnotetext{
${ }^{1}$ Université de Lorraine, CREM, France.

${ }^{2}$ Notre traduction. La citation originelle est : «Everything crumbles. Possum Springs bleeds to death. And soon we'll all be dead and this town will just be fields and trees. »
} 
Jacques Henriot notait que « le jeu prend modèle sur une réalité qui, à son tour, pour mieux se faire comprendre, le prend pour modèle et parle son langage. Du modèle en vraie grandeur au modèle réduit, de la chose à l'image s'accomplit un incessant va-etvient » (Henriot, 1989 : 61). C'est d'une de ces réalités dont il est question dans Night In The Woods (Infinity Fall, 2017)3. La famille de Mae Borowski - avatar du joueur - est présentée comme une famille étasunienne moyenne d'une petite ville minière peuplée d'animaux anthropomorphes. Afin de permettre à leur fille d'aller à l'université, cette famille a dû s'endetter. Dans le jeu, il est question, entre autres, de la saisie de leur maison par la banque avec laquelle ils ont contracté un prêt, de la reconversion professionnelle du père et du suivi budgétaire familial que fait la mère. Au cours de sa partie, le joueur peut révéler les problèmes économiques de la famille Borowski et des habitants de cette petite ville en discutant avec l'ensemble des personnages. En effet, les citoyens que rencontre le joueur ou la joueuse font tous et toutes face à des problèmes existentiels plus ou moins liés à la récession économique. Par exemple, Beatrice, amie de Mae et dont le père est chômeur de longue durée, n’a pu poursuivre ses études à l'université. Bien que ce monde soit fictif, il est difficile de ne pas y voir de nombreuses références aux États-Unis des années 2000 à 2010.

Si l'année 2008 a été emblématique pour le jeu vidéo indépendant avec les sorties de Braid (Number None) et de Word Of Goo (2D Boy), elle a aussi marqué les esprits pour avoir été la seconde partie de la crise des subprimes. Jean-Charles Bricongne, Vincent Lapègue et Olivier Monso notèrent que « des ménages américains dont l'accès au crédit était jusque-là très limité, parce qu'ils ne présentaient pas de garantie suffisante en termes de revenu, d'emploi ou de patrimoine, se sont vu offrir la possibilité de contracter des emprunts immobiliers mieux adaptés à leurs besoins » (Bricongne et al., 2009 : 26). Ce contexte a contribué à l'émergence de récits en lien avec des problématiques économiques et sociales proches des expériences de vie des créateur·ice-s et des joueur·euse-s. La réflexion que nous proposons est double. Premièrement, nous souhaitons apporter des éléments de réponse à la question suivante : comment analyser et qualifier la persuasivité des discours à propos d'enjeux sociétaux contenus dans un jeu vidéo ? Secondement, comment analyser les stratégies employées par les game designers afin de transmettre aux joueuses et joueurs une représentation, parcellaire, de leurs opinions ? Pour cela, nous mobiliserons une étude de cas particulière : les discours portés par NITW sur la situation économique des ménages habitant la Rust Belt $t^{4}$.

Si l'expressivité fait référence à ce qui est expressif, nous définissons la persuasivité comme le caractère de ce qui est persuasif. Ce concept fait donc référence à l'ensemble des stratégies discursives de persuasion contenues dans un jeu. La question de la persuasivité a déjà été abordée de plusieurs façons au sein des game studies. Ian Bogost définit, en 2007 (Bogost, 2007), les persuasive games comme des jeux dont l'intention est de convaincre leur joueur d'un message qu'ils contiennent. Il s'appuie alors sur la

\footnotetext{
${ }^{3}$ Abrégé NITW par la suite.

${ }^{4}$ La Rust Belt correspond à la région entourant les Grands Lacs : les États de New York, Pennsylvanie, Virginie de l'ouest, I'Ohio, I'Indiana, le Michigan, I'Illinois, l'lowa et le Wisconsin.
} 
notion de rhétorique procédurale, issue de procédures informatiques, pour attribuer une spécificité propre au média vidéoludique. Plusieurs critiques ont porté sur l’importance des représentations issues des assets ${ }^{5}$ et autres éléments non interactifs pour ce qui est du décodage du discours, notamment celle de Doris Rusch qui évoque les limites de «l'expression procédurale » lorsqu'il s'agit d'enjeux personnels (Rusch, 2009). Elle met particulièrement en avant l'importance des « métaphores expérientielles » dans le but de lier l'expérience que le ou la joueuse a en jouant et les expériences qu'il ou elle peut avoir dans sa vie personnelle.

Afin de prendre en compte les critiques adressées aux persuasive games, nous proposerons un cadre conceptuel notamment issu du diagramme de ludicisation ${ }^{6}$ de Sébastien Genvo (Genvo, 2013) et de la modélisation de la narration vidéoludique ${ }^{7}$ proposée par Rémi Cayatte (Cayatte, 2018). Nous optons pour ces modélisations principalement pour le fait qu'elles s'attachent à représenter l'objet support du - ou des - discours (le système de règles et l'éthos vidéoludique chez Genvo ; l'expérience-cadre chez Cayatte), le joueur ou la joueuse (le joueur-modèle pour le premier et les procédures pour le second) et les interactions entre ces acteurs. Nous les compléterons en mobilisant la cybernétique et les sciences de l'éducation en plus des game studies pour analyser les situations de communications rencontrées dans un jeu vidéo.

Pour cet article, NITW a été parcouru de deux façons. Premièrement en ligne droite : nous avons suivi la trame principale du jeu sans nous préoccuper des récits annexes. NITW est un jeu mettant l'emphase sur les discussions que Mae peut avoir avec les habitants de Possum Springs : narration par des dialogues, textes multiples sur la ville, etc. Lors du second parcours du jeu, nous avons donc systématiquement discuté avec l'intégralité des $\mathrm{PNJs}^{8}$ lors des temps d'exploration de sorte à révéler l'ensemble des récits annexes. Dans le but de dévoiler le plus possible l'histoire de la ville, nous avons systématiquement opté pour les lignes de dialogue entrainant une réponse explicative de la part du ou des PNJs participants (inférence, questions, etc.).

\section{Plusieurs moments de jeu pour soutenir une pluralité de discours}

NITW démarre lorsque Mae arrive à la gare de Possum Springs. Après une traversée dans les bois, elle parvient au domicile familial et y trouve son père devant la télévision, surpris de sa venue. Après une première nuit passée, Mae peut alors partir à la (re)découverte de sa ville d'origine, et ce durant les deux semaines qui constituent le récit. Ce dernier est séparé en quatre actes ayant un nombre de jours variable. Son organisation est relativement cadrée : un acte est composé de plusieurs journées. Ces dernières sont

\footnotetext{
${ }^{5}$ Tout élément visuel ou sonore d'un jeu vidéo, un asset peut lui-même être le produit d'autres assets.

${ }^{6} \mathrm{Cf}$. Annexe 1.

${ }^{7} \mathrm{Cf}$. Annexe 2.

8 Personnages Non-Joueur.euse·s.
} 
alors séquencées chronologiquement. Généralement, une journée se décompose ellemême en deux temps.

Le premier temps laisse le joueur ou la joueuse libre (1) d'explorer l'ensemble des quartiers accessibles de la ville, notamment pour discuter avec l'ensemble des habitants et révéler les récits annexes ou (2) de se rendre directement auprès de Gregg, Beatrice ou Angus, amis de Mae et personnages principaux dont les fonctions en termes de mécaniques de jeu à ce moment sont de poursuivre le récit principal. Durant ces périodes, il y a donc une double exploration. La première est spatiale et consiste à traverser la ville de Possum Springs composée de « signes spatiaux naturels » et de «signes spatiaux fonctionnels ( (Grandjean, 2019). « Les signes spatiaux naturels ne remplissent aucune fonction autre que phénoménologique »(Grandjean, 2019), il s'agit donc d'éléments permettant de susciter chez le joueur ou la joueuse la sensation qu'il ou elle explore une ville de la Rust Belt. Par exemple, on peut apercevoir au loin des anciennes usines aujourd'hui clôturées. En traversant la ville, le joueur ou la joueuse passe devant ce qui reste, au moment du récit, des boutiques ayant fermé auparavant. En revanche, les " signes spatiaux fonctionnels » permettent au joueur ou à la joueuse de poursuivre soit le récit principal du jeu, soit l'un des multiples récits annexes en induisant une seconde forme d'exploration qui est, cette fois, plutôt hypertextuelle. Outre son aspect plateforme et les mini-jeux 9 qu'il contient, le titre propose à son joueur ou sa joueuse de nombreuses discussions (dont certaines durant lesquelles il ou elle ne fait qu'écouter et peut partir à tout moment) permettant de révéler progressivement les sujets d'actualités et les problèmes de chacun·e des habitant·e·s de Possum Springs (figure $n^{\circ} 1$ ). Occasionnellement, le fait de discuter avec l'un·e des habitant.e-s peut aboutir à une séquence dirigiste durant laquelle le joueur ou la joueuse est guidéee par le game design à effectuer certaines actions précises : casser des biens publics, explorer les égouts de la ville, rechercher des constellations, etc. Certaines discussions peuvent se révéler triviales (le joueur ou la joueuse peut par exemple discuter avec Selmers pour entendre chaque jour de nouveaux poèmes) alors que d'autres peuvent toucher des problématiques plus personnelles à chacun des PNJ. Lors d'une discussion avec Stan, le père de Mae, le joueur ou la joueuse découvre les difficultés qu'il a eues à trouver son emploi actuel de boucher au Ham Panther (la grande surface de Possum Springs) et à quel point il le déteste ${ }^{10}$. Pendant ce temps, il est donc principalement question d'explorer et de révéler les thématiques abordées par le jeu. Hormis lors des rencontres avec Gregg, Angus ou Beatrice, le joueur ou la joueuse n'est jamais tenu.e d'interagir avec chacun des PNJ qui sont des « signes spatiaux fonctionnels ». Au sens de Nicolas Auray et Bruno Vétel, il s'agit donc de considérer ce temps comme un moment propice à l'ouverture attentionnelle curieuse :

\footnotetext{
9 Il est notamment question, en termes de mini-jeux, de tir sur une cible, d'actions contextuelles reprenant les jeux Guitar Hero (Harmonix Music System, 2005), etc.

${ }^{10}$ Stan reconnait cependant que son travail est meilleur pour son dos. Sans mentionner la problématique, le jeu aborde tout de même la question de la pénibilité du travail.
} 


\begin{abstract}
À la différence d'un simple contrôle de l'activité qui tente de résorber les imprévus venus du contexte, l'ouverture attentionnelle curieuse, que l'on peut aussi appeler « agir exploratoire », consiste à se rendre disponible à des perturbations, à ne pas les traiter comme des interruptions (qui créeraient une discontinuité dans l'action), mais à ne pas non plus les envisager comme des dispersions (qui créeraient une simple versatilité de la personne au gré des situations, sans guidage) (Auray et Vétel, 2013 : 159).
\end{abstract}

Le second temps composant une journée du récit consiste cette fois en une séquence généralement plus dirigiste : soit parce qu'elle a lieu dans un environnement restreint, soit parce que le joueur ou la joueuse est limitée dans le choix des actions qu'il ou elle peut réaliser. Le joueur ou la joueuse est plus ou moins guidée afin de minimiser les ruptures dans l'action. Par exemple, la première séquence que rencontre le joueur ou la joueuse consiste en une soirée song and pizza du groupe d'amis de Mae (Gregg, Angus, Beatrice et parfois Germ) : une répétition musicale suivie d'un repas au Clik Clak Diner, un restaurant dans le centre-ville de Possum Springs. C'est après ce repas qu'un élément central de l'intrigue est révélé, puisque le groupe découvre dans la rue un bras en décomposition et tatoué. Contrairement au premier temps que nous avons décrit, il s'agit donc ici de faire progresser le joueur ou la joueuse sans que celle-ci ne puisse flâner. C'est aussi lors de ces moments que l'on peut lire des opinions sur la situation économique de la ville. Plus tard dans le jeu, Mae et Beatrice vont au centre commercial Fort Lucenne. Il s'agit là d'un second temps d'une journée typique de NITW. À leur arrivée, Mae est surprise par l'absence de clientèle et par les nombreux magasins fermés par manque d'activité. Bea explique cette perte de vitesse de la galerie marchande en accusant Internet ${ }^{11}$. Le jeu propose une illustration de ce que Nora Aufreiter et al. nomment notamment le retail store apocalypse, c'est-à-dire la fermeture des boutiques et l'abandon des galeries marchandes laissées en friche (Aufreiter et al., 2012).

La narration proposée par NITW correspond à cette alternance de moments propices à l'ouverture attentionnelle curieuse. Elle permet de révéler l'histoire de la ville et les récits de vie de chacun des habitants. Mae peut discuter avec ces derniers durant des moments plus linéaires mais approfondissant les connaissances que les joueurs et les joueuses peuvent avoir sur la ville et ses habitants.

\footnotetext{
${ }^{11}$ Et par métonymie les sites de commerce tels qu'Amazon.
} 


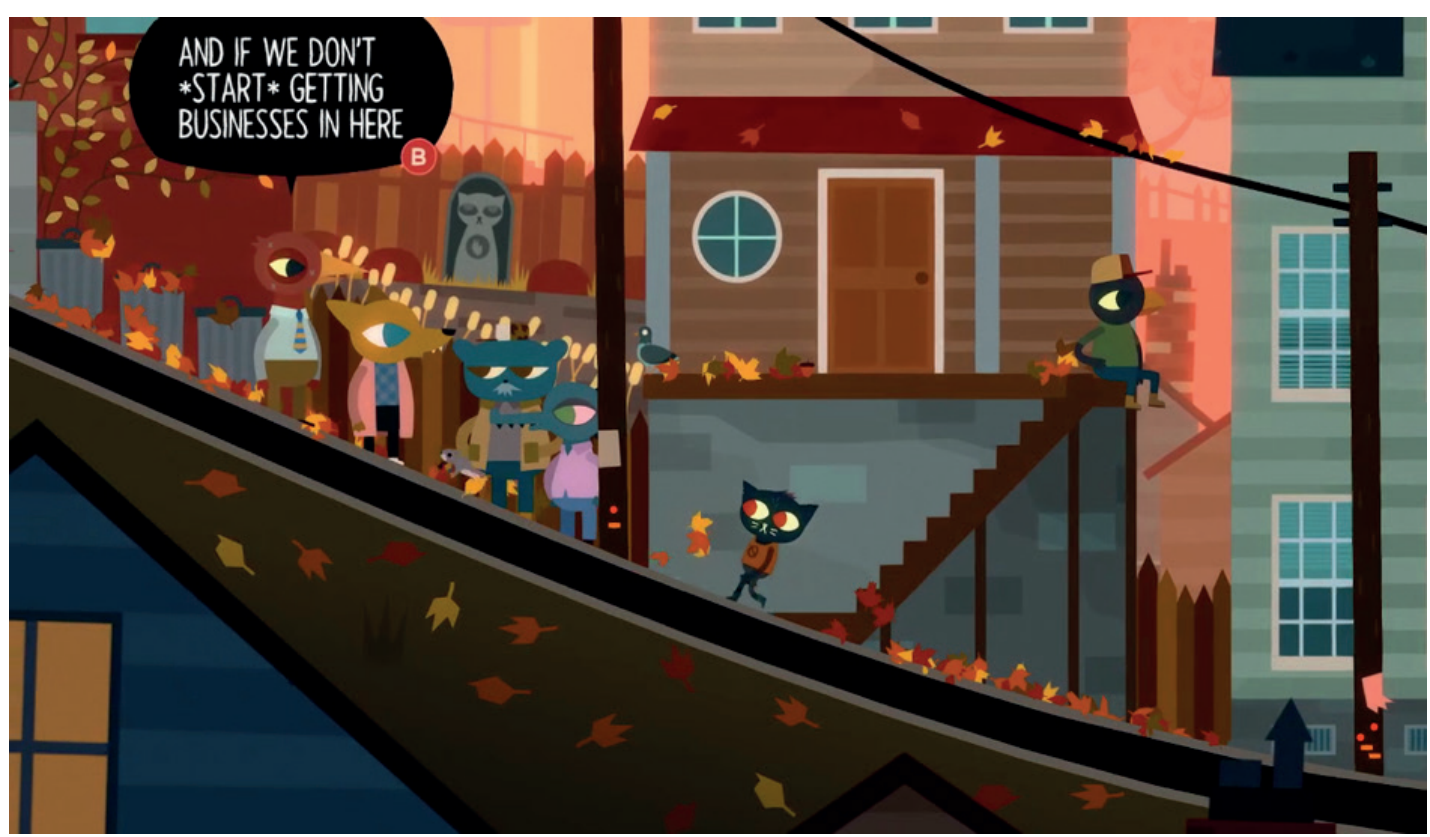

Figure N 1 : Discussion ENTRE LES MEMBRES DU CONSEIL DE VILLE (NITW, 2017)

Ce séquençage en deux temps que nous avons présenté fait apparaitre plusieurs moments de jeux qui présentent des gameplays différents et de fait, des figures de rhétorique hétéroclites. Il apparait alors nécessaire de catégoriser ces moments afin de définir la persuasivité d'un jeu. Plusieurs auteurs et autrices ont déjà proposé de discuter cette notion de " moments » dans un jeu vidéo, soit de manière directe (Giner, 2017) en faisant la distinction entre une situation et un moment (le dernier naît de la première s'il y a conscientisation), soit en analysant le comportement du joueur ou de la joueuse lorsqu'il ou elle fait notamment des allers et retours entre phases de jeu et phases réflexives (Sohier, 2016). La critique principale que nous effectuons vis-à-vis de ces propositions concerne l'importance accordée à ce qui est relatif au play ${ }^{12}$, aux joueurs et joueuses et donc aux procédures (au sens de Cayatte, 2018), sans accorder d'attention particulière au jeu vidéo en tant qu'objet cybernétique. À l'instar d'Alexander R. Galloway,

[we]embrace the claim, rooted in cybernetics and information technology, that an active medium is one whose very materiality moves and restructures itself-pixels turning on and off, bits shifting in hardware registers, disks spinning up and spinning down (Galloway, 2006 : 18).

Galloway compte quatre gamic actions qui permettent de décrire le moment de jeu. Son objectif est de décrire la relation cybernétique entre un opérateur-joueur et la machine lorsqu'une session de jeu est effective. Sa proposition permet de considérer le jeu vidéo comme étant une forme de communication entre (1) une machine appliquant les ordres qu'elle reçoit d'un code informatique écrit par des développeurs et des développeuses et (2) un opérateur qui produit un ensemble d’inputs et de procédures en

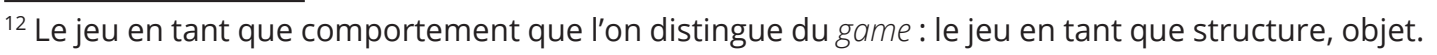


fonction des ordres et des contraintes fournis par le jeu. Galloway propose de distinguer ces actions vidéoludiques en fonction de leur caractère diégétique ou non-diégétique ${ }^{13}$ mais aussi en fonction de l'origine de ces actions : soit l'opérateur, soit la machine. Il est possible de représenter cette réflexion sous la forme d'un tableau à double entrée (tableau $\mathrm{n}^{\circ}$ 1). Quatre formes d'actions définies par Galloway apparaissent alors : play, process, algorithm et code.

Tableau 1 : les Quatre types d'actions vidéoludiques selon Galloway.

\begin{tabular}{|l|l|l|}
\hline \multirow{2}{*}{ Diégétique } & L'opérateur & La machine \\
\cline { 2 - 3 } & Play & $\begin{array}{l}\text { Process } \\
\text { rendre visite à Gregg. }\end{array}$ \\
\hline \multirow{2}{*}{ Non diégétique } & \begin{tabular}{l} 
Algorithm \\
\cline { 2 - 3 }
\end{tabular} & $\begin{array}{l}\text { Ex : accélérer certaines discus- } \\
\text { Possum Springs. }\end{array}$ \\
\cline { 2 - 3 } & $\begin{array}{l}\text { sions des passants sans y } \\
\text { prendre part. }\end{array}$ & $\begin{array}{l}\text { Ex : les phylactères contenant les } \\
\text { lignes de dialogue qui se } \\
\text { déroulent automatiquement. }\end{array}$ \\
\hline
\end{tabular}

Les exemples reposent sur la figure $n^{\circ} 1$.

À partir de ce tableau, il nous semble possible de proposer un premier paradigme dans le but de modéliser la persuasivité d'un discours porté par un jeu vidéo. Nous proposons de définir cette dernière sous la forme d'un continuum persuasif dont chacune des étapes est fonction des controverses générées par la simultanéité des actions vidéoludiques lors d'une situation-séquence ${ }^{14}$ de jeu. Nous définissons ici les controverses comme des phénomènes de communication entre l'opérateur et la machine qui permettent la co-construction des discours. À l'issue de nos parcours de NITW, nous remarquons que celui-ci met principalement l'emphase sur le play, le code et dans une moindre mesure les process. Les controverses que nous observons se concentrent particulièrement entre ce qui relève du play et ce qui relève du code. Si le premier temps de la journée dans NITW laisse s'exprimer l'opérateur via son exploration de la ville, les actions de code restent présentes lors des discussions et des mini-jeux. C'est fondamentalement l'opérateur, par le play et son « agir exploratoire »"15 qui va les déclencher. On peut donc énoncer que les discours, bien que présents à ce moment, ne sont pas imposés aux joueurs et joueuses. Inversement, les seconds temps des journées sont bien plus dirigistes du fait des zones explorables ou par ce que peut faire le ou la joueuse : le play est donc contraint (principalement par mini-jeux ou par le choix de certaines

\footnotetext{
${ }^{13}$ Nous préférons ce terme qui englobe l'extradiégétique qui relève du jeu (écran de chargement, inventaire, menus, etc.) mais aussi ce qui relève de la machine (puissance de calcul, système d'exploitation, etc.).

${ }^{14}$ Nous optons pour l'appellation de situation-séquence puisque ce mot-valise englobe formellement un lieu (la situation) avec une temporalité (la séquence).

15 Nous interprétons «l'agir exploratoire » comme étant une posture que le joueur adopte dans sa façon de jouer. Nous reviendrons par la suite sur la façon dont nous proposons de prendre en compte cela pour analyser la persuasivité des discours.
} 
lignes de dialogues de Mae). Durant ces parties du jeu, nous énonçons que c'est plutôt le code, donc une action machinique et non-diégétique, qui conditionne et enclenche le play du joueur.

Cependant, pour les façons dont nous analysons les deux temps du jeu à partir des actions vidéoludiques de Galloway, il convient de dire que nous pourrions proposer une analyse plus précise en catégorisant les moments de jeu. Il devient donc nécessaire de qualifier les controverses entre les actions de l'opérateur et celles de la machine et donc de définir la forme que prend la communication effective entre les acteurs d'une situation-séquence de jeu. Dans la suite de cet article, nous explorons la façon dont il est possible de représenter ces formes de communication, et ce dans le but de qualifier la persuasivité d'un discours contenu dans un jeu vidéo, en l'occurrence le discours concernant la situation économique des petites villes de la Rust Belt dans NITW.

\section{Analyser et qualifier la persuasivité des jeux vidéo}

Possum Springs est une représentation des shrinking cities avec, certes, des animaux anthropomorphes pour habitants. Celles-ci sont des « villes "marginalisées" par rapport au capitalisme, fonctionnant comme des espaces périphériques dans les circuits du capital » (Béal et al., 2016 : 232). Possum Springs est une ancienne ville minière. Il est notamment possible de distinguer les anciens locaux d'une usine ${ }^{16}$ dans le décor du jeu. L'ancien métro conduisait directement les habitants à l'usine, signifiant que la ville était probablement son bassin d'emplois. Il n'est pas rare de croiser des habitants PNJs regrettant la gloire passée de la ville lorsque la région portait encore le nom de «Manufacturing Belt $»^{17}$. Si la catégorie du post-apocalyptique ne convient pas à NITW, le jeu décrit néanmoins une ville frappée par la récession économique. Son level design ${ }^{18}$ et sa modélisation opèrent comme « métaphore expérientielle ». En ce sens, nous rejoignons la conclusion de Laurent Di Filippo et Patrick Schmoll sur la mobilisation d'un décor urbain :

Le décor urbain, pour sa part, a au moins deux utilités : d'un côté, il fait office de symbole du monde moderne et, de l'autre, il sert de référence au quotidien des joueurs. Les relations qui se tissent entre la ville physique actuelle et l'activité ludique sont au cœur des processus de transformation et des nouvelles appropriations possibles (Di Filippo et Schmoll, 2016 : 133).

Ainsi, le choix d'ancrer le récit de NITW dans une ville aisément identifiable n'est pas anodin. Durant les phases d'exploration, le joueur dispose d'une certaine liberté pour révéler les propos contradictoires de ses habitants. Un exemple de ces contradictions est notamment observable à la fin du récit lorsque le jeu offre une lueur d'espoir au marasme ambiant en illustrant, dans ses derniers instants, l'ouverture d'une nouvelle enseigne dans le centre-ville. Par ailleurs, Mae est présentée comme une personne ni-

\footnotetext{
${ }^{16}$ Que nous supposons être la verrerie dans laquelle Stan, le père de Mae, a travaillé avant sa période de chômage.

${ }^{17}$ C'est ainsi que la Rust Belt est nommée jusque dans les années 1970.

${ }^{18}$ Que nous définissons comme la façon d'agencer les zones explorables d'un jeu.
} 
hiliste, cette attitude la pousse à ne jamais prendre fondamentalement parti sur les opinions des habitants à propos de la situation économique de la ville. Il s'agit là aussi de permettre aux joueurs et joueuses d'imaginer les pensées de Mae et de finalement décider de ses propos. Nous rejoignons donc Fanny Barnabé et Julie Delbouille qui énoncent : « en servant de médiation entre le joueur et l'objet jeu, l'avatar est l'un des principaux vecteurs de [la] réflexivité intrinsèque des œuvres vidéoludiques » (Barnabé et Delbouille, $2018: 4$ ).

Encadré 1 : Propos tenus par le leader du Culte sévissant à Possum Springs lors d'une situation-séquence mettant l'emphase sur le code plutôt que le play

Things *can* get better. These mines are gonna be humming again someday. Old mills puffin smoke. You kids don't understand that - we lost what our world was built around. Used to be you provided for a family, bought a house. Now you're stockin shelves at the grocery store. Kids leavin' more than they're stayin'... No opportunity here. Old people dyin', houses left empty, you ever seen that? A * home* become a tumbled-in pile of wood and plaster? A *job* become a burned out brick box or a hole in the ground? But we can change that. We can put this place back together.

D’une manière générale, le jeu n'oblige pas le joueur ou la joueuse à révéler l'ensemble des discours, des situations de vies et des expériences relatées par chacun des habitants de la ville. Certains sont inévitables (comme celui de l'encadré $\mathrm{n}^{\circ}{ }^{1}$ ) et d'autres dépendent de l'attitude du joueur et donc de son ouverture attentionnelle curieuse. Nous concluons donc que le game design de NITW alterne des séquences persuasives et des séquences expressives. Genvo propose la notion d'expressive games pour définir des jeux souhaitant installer une relation empathique sans volonté persuasive (2016). Cependant, nous adressons à ces deux genres vidéoludiques - les expressive games et les persuasive games - la même critique que Gabrielle Trépanier-Jobin (2016) : il s'agit de deux concepts (1) se rapportant à l'éthos des jeux - la façon dont sont présentés les discours au joueur - et (2) se référant plutôt aux intentions de leurs auteurs, notamment celles transmises en dehors des jeux par un ensemble de paratextes (textes de présentation, publicités, entretiens avec les auteurs, etc.) qui composent alors l'éthos prédiscursif du jeu ${ }^{19}$. Ainsi, le parallèle entre Possum Springs et la Rust Belt est potentiellement plus évident dans les témoignages des auteurs que dans le jeu :

College dropout Mae Borowski returns home to the crumbling former mining town of Possum Springs seeking to resume her aimless former life and reconnect with the friends she left behind (NITW sur Steam, s. d.) $)^{20}$.

Creators of the games said they were more interested in showing the complicated lives of the people and places the world has left behind, as well as the economic realities that inevitably circumscribe their stories (Hudson, 2017 : B7).

Avec NITW, nous sommes donc loin de la représentation idyllique que faisait Bogost en 2008 à propos des problèmes budgétaires du joueur dans Animal Crossing (Nintendo,

\footnotetext{
${ }^{19}$ C'est-à-dire l'ensemble des éléments annonçant le contenu et les propos du jeu.

20 NITW sur Steam. (s. d.). Consulté le 17 décembre 2018, à l'adresse : https://store.steampowered. com/app/481510/Night_in_the_Woods/
} 
2002), réduits à des choix de mobiliers. À l'instar de Cart Life (Hofmeier, 2010) dont le protagoniste est un vendeur précaire de journaux, NITW représente une société après la crise économique de 2008 dans laquelle les ménages moyens ont des difficultés à subvenir à leurs besoins. Dans un entretien donné au New-York Times, Benson énonce:

"We want to create stories and mythologies about the places we're from and the people we know, and that includes addressing the economics of it," said Mr. Benson, one of the NITW developers. "If you don't, I think you're not getting the whole picture" (Hudson, 2017: B7).

À partir de ces quelques paratextes, il est possible d'inférer les éthos discursifs (sensibles au cours du jeu) et prédiscursifs (précédant le jeu et notamment relatifs aux paratextes) de l'expérience-cadre (au sens de Cayatte, 2018). Pour NITW, il semble variable en fonction des récits incorporés ${ }^{21}$ dans l'expérience-cadre et des paratextes observés. Cependant, nous discutons la temporalité supposée entre ce qui est discursif et le prédiscursif. Ruth Amossy avance : « tout éthos discursif se construit sur la base d'un éthos préalable, ou prédiscursif » (2014 : 23). L'enjeu n'est pas de confronter ses propos mais plutôt de modéliser la simultanéité de ces deux éthos. Ils participent aux discours du jeu et à l'adoption de certaines procédures de la part du ou de la joueuse. Inversement, le ou la joueuse participe à la aussi à la construction de ces discours : (1) en respectant l'expérience-cadre ou en la détournant et (2) en produisant des paratextes ou en validant ou rejetant des systèmes de représentation issus d'autres paratextes (figure $\mathrm{n}^{\circ} 2$ ).

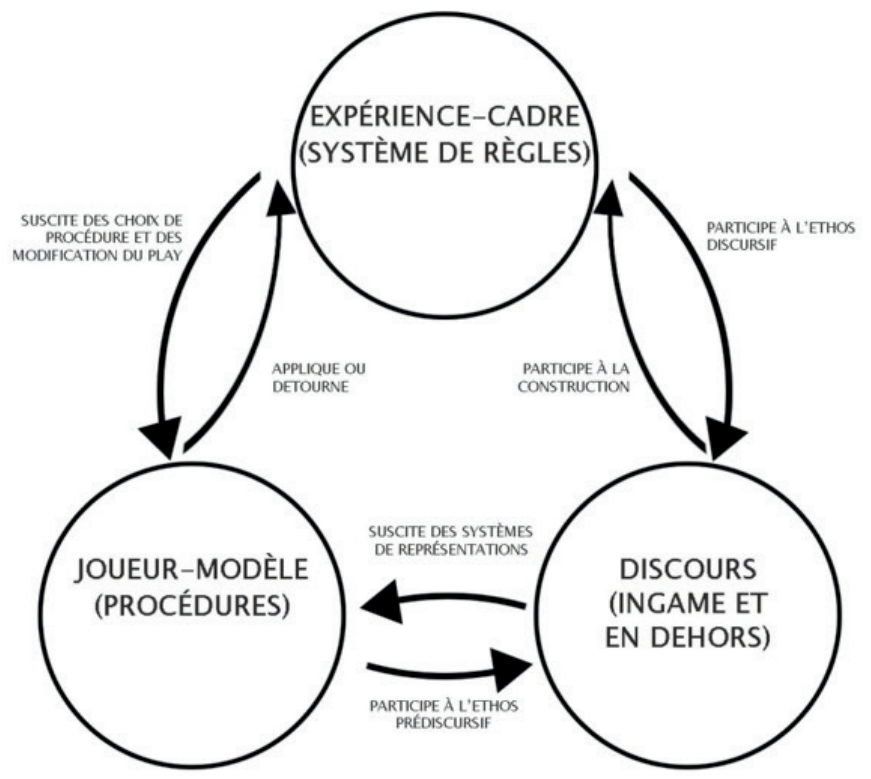

FIGURE N ${ }^{\circ} 2$ : LeS RELATIONS ENTRE L'EXPÉRIENCE-CADRE, LE JOUEUR-MOdèLE ET LES DISCOURS

Par le biais du modèle représenté en figure $\mathrm{n}^{\circ} 2$, nous représentons la façon dont des discours contenus dans un jeu peuvent être plus ou moins communiqués aux joueurs

${ }^{21}$ En référence à Cayatte (2018). 
et joueuses. Cette représentation s'inspire en partie du diagramme de ludicisation de Genvo et schématise une situation potentielle durant laquelle un joueur peut être l'audience d'un discours (voir annexe 1). L'adjectif «potentielle » est particulièrement important puisque les joueurs peuvent ne pas prêter attention aux discours contenus dans une situation-séquence ainsi qu'à ce qui entoure le jeu tels que les différents paratextes que nous avons présentés. Par ailleurs, l'intérêt de la figure $\mathrm{n}^{\circ} 2$ est qu'elle est proche visuellement et conceptuellement de certains cadres théoriques issus des sciences de l'éducation. En particulier, elle adapte le triangle pédagogique de Jean Houssaye (Houssaye, 1993)22 à la façon dont les discours ingame et en dehors émergent. Ce rapprochement illustre aussi les relations que ces discours (les savoirs, ibid.) ont avec l'expérience-cadre (assimilé à « l'enseignant », ibid.) et le joueur-modèle (l'étudiant, ibid.). Cependant, plutôt que de considérer les discours comme des entités préexistantes, autonomes et distinctes - c'est le cas des savoirs chez Houssaye - nous énonçons qu'ils sont le résultat de controverses entre les procédures (par les actions du joueur ou de la joueuse) et l'expérience-cadre (contenant des éléments de discours). C'est pourquoi nous nous focalisons sur celle-ci. Pour cela, nous proposons de mobiliser le modèle des événements d'apprentissages - enseignements de Dieudonné Leclercq et Marianne Poumay (2008) afin de qualifier la communication se déroulant lors du gameplay selon Cayatte (annexe 2).

Â travers ce prisme, nous concevons le gameplay de NITW comme un ensemble de situations-séquences pédagogiques potentielles durant lesquelles plusieurs événements d'apprentissages-enseignements ${ }^{23}$ à propos de l'après-crise peuvent survenir. Ces événements sont caractérisés par un ensemble d’objectifs communicationnels, d'éléments ingame (des assets diégétiques ou extra diégétiques) et des approches pédagogiques (apprentissage par répétition, par exploration, par expérimentation, etc.). Ces EAE ont l'intérêt de prendre en compte l'ensemble des acteurs d'une communication. Bien que le concept des EAE soit originellement mobilisé pour définir les événements ayant lieu entre un formateur et un apprenant, nous l'appliquons au jeu en tant que situation de communication avec un joueur ou une joueuse déployant des procédures selon des attitudes et des comportements particuliers. Dès lors, il s'agit de qualifier les approches du game design (les actions vidéoludiques réalisées par la machine) et les comportements du joueur (les actions vidéoludiques réalisées par l’opérateur) plus ou moins attendus (tableau $\mathrm{n}^{\circ} 2$ ).

\footnotetext{
${ }^{22}$ Houssaye définit le triangle pédagogique comme un espace compris entre le savoir, l'enseignant et l'étudiant. Par ailleurs, il définit sous l'appellation de « postures » des relations privilégiées entre ces acteurs, idée que nous ne retenons pas ici mais qui pourra faire l'objet d'un prochain travail. 23 (Abrégé EAE).
} 
TABleau N ${ }^{\circ} 2$ : Les ÉVÉNEMENTS D'APPRENTISSAGES-ENSEIGNEMENT APPLIQUÉS À UNE SITUATION-SÉQUENCE VIDÉOLUDIQUE

\begin{tabular}{|c|c|c|}
\hline $\begin{array}{c}\text { Les approches de } \\
\text { l'expérience-cadre } \\
\text { Process et Code }\end{array}$ & $\begin{array}{c}\text { Les procédures attendues } \\
\text { de la part du joueur-modèle } \\
\text { Play et Algorithm }\end{array}$ & \multirow{2}{*}{ Continuum Persuasif } \\
\hline Guidance & Exercisation & \\
\hline Modélisation & Observation & \multirow{2}{*}{} \\
\hline Transmission & Réception & \multirow{2}{*}{ Expressif } \\
\hline Animation & Débat \\
\hline Réactivité & Expérimentation \\
\hline Confortation & Création \\
\hline Documentation & Exploration & \\
\hline Co-réflexion & Méta-réflexion & \\
\hline
\end{tabular}

Les approches et les procédures se basent sur le modèle des EAE de Leclerc et Poumay (2008).

À partir de cette grille de lecture, il devient possible de catégoriser la persuasivité des discours contenus dans NITW. Nous énonçons que plus une situation-séquence comprend des EAE tendant vers la persuasivité et plus le game design sera persuasif. Inversement, plus le game design proposera des situations-séquences laissant le joueur contrôler son apprentissage et plus le jeu sera expressif à propos d'un discours qu'il contient. Par exemple, NITW dans son ensemble ne propose pas d'événements [guidance - exercisation] hormis pour les mini-jeux DemonTower ${ }^{24}$ et musicaux : Mae n'est jamais invitée à « réessayer » après ce qui peut sembler être un échec. Il n'y a pas non plus de [confortation - création] ni de [réactivité - expérimentation] puisque le ou la joueuse n'est pas libre de tester ou de créer des éléments à partir de l'expérience-cadre. Malgré les quelques choix de dialogues, il n'y a pas non plus d'invitation à susciter un débat entre des participants.

En revanche et à propos des shrinking cities, le game design ${ }^{25}$ de NITW transmet des éléments de discours variés à propos de la situation économique de Possum Springs en insérant dans les situations-séquences (et donc le gameplay) les EAE suivants :

- [Modélisation - observation] : le joueur ou la joueuse a le loisir d'observer chacun des assets composant la ville de Possum Springs. Cet EAE ne repose pas sur un objectif formalisé dans le jeu, c'est donc le play qui est à l'initiative ;

- [Transmission - réception] : l'intégralité de la narration vidéoludique passe par des textes mais le joueur ou la joueuse n'est pas obligé de recevoir l'entièreté de ces textes (les récits secondaires) pour faire avancer le récit. Le play tient donc une part importante du déclenchement de la transmission. Cependant, la trame principale

\footnotetext{
${ }^{24}$ Un jeu singeant la série Dark Souls.

${ }^{25}$ Et donc les auteurs et autrices.
} 
ne permet pas au joueur ou à la joueuse de faire des ellipses, c'est donc la machine par le biais du code (pour reprendre Galloway) qui est à l'initiative ;

- [Documentation - exploration] : le code documente la situation de la ville et de ses habitants par des lignes de dialogues et des éléments révélant l'histoire. C'est au joueur ou à la joueuse d'aller à la rencontre de cette documentation en explorant la ville. C'est donc le play qui est à l'initiative ;

- [Co-réflexion - Méta-réflexion] : par l'ensemble des textes et des assets, le game design apporte des éléments de discours à propos de la situation économique de Possum Springs en tant que shrinking cities (tels que des opinions, des coupures de presse relatant l'histoire de la ville, etc.). Ceux-ci peuvent servir aux joueurs et joueuses afin qu'ils et elles puissent développer leurs réflexions en prenant en compte l'importance des métaphores expérientielles (Rusch, 2008) et des contextes dans lesquels ils et elles jouent (Genvo, 2013).

À partir de la méthode que nous avons exposée, il nous semble possible d'amener des preuves formalisées de la persuasivité d'un jeu, en l'occurrence NITW. Plutôt que de le catégoriser d'un seul tenant en tant que persuasive game ou d'expressive game, il est judicieux d'exercer une lecture plus détaillée. Sur quatre des EAE retenus pour définir le gameplay de NITW, il apparait que seuls les EAE de [transmission - réception] rencontrés lors de la trame principale du jeu sont incontournables dans le parcours du joueur ou de la joueuse. Par ailleurs, une partie de ces EAE (lors du premier temps décrit d'une journée ingame) n'est activable que par l'exploration facultative du joueur ou de la joueuse. Les trois autres sont à l'initiative du joueur ou de la joueuse : si ce ou cette dernière adopte une posture d'ouverture attentionnelle curieuse, il ou elle a accès à de nombreux éléments. Enfin, il convient de prendre en compte la pluralité des opinions diffusées qui teintent les dialogues obligatoires et facultatifs du jeu. Malgré l'omniprésence des discours à propos de la situation économique de Possum Springs et malgré les paratextes que nous avons mobilisés et ceux restants, nous énonçons que sur le point précis de la situation des petites villes (et plus précisément des shrinking cities) de la Rust Belt, le jeu est expressif. Cependant, il est important de mentionner qu'il peut être persuasif concernant d'autres sujets. Par exemple, nous n'avons pas traité dans cet article de la dépression de Mae. Il pourra être intéressant dans un prochain travail de pratiquer un examen similaire avec la méthode que nous proposons afin de déterminer si le jeu est expressif ou persuasif à ce sujet.

\section{Définir la persuasivité des discours vidéoludiques en mobilisant les sciences de l'éducation}

L'objet de cet article était double. Premièrement, nous souhaitions proposer un modèle permettant d'analyser la persuasivité ou l'expressivité d'un jeu vidéo à propos d'un discours qu'il contient et qui émerge avec l'intervention du joueur ou de la joueuse. Secondement, en faisant de NITW notre étude de cas, nous voulions observer les mé- 
thodes des game designers dans la création d'une expérience-cadre portant un discours spécifique. En l'occurrence, les enjeux de ces observations portaient sur la façon dont NITW porte un discours plutôt expressif composé d'éléments parfois contradictoires sur les shrinking cities de la Rust Belt. Sans énoncer qu'il y aurait une spécificité du jeu vidéo indépendant à être expressif, NITW semble paradigmatique dans la façon dont son game design structure la communication entre ses auteur.rice.s et le joueur ou la joueuse : l'objectif est plutôt de le ou la laisser autonome dans ce qu'il ou elle peut apprendre à propos des villes étasuniennes similaires à Possum Springs.

Nous concluons sur l'intérêt de mobiliser les outils de la pédagogie et des sciences de l'éducation pour non pas inférer les potentiels pédagogiques des jeux vidéo mais pour mettre en place une méthodologie des études de discours ingame. Le cadre que nous proposons permet alors de qualifier la persuasivité de ces phénomènes de communication. En complétant le modèle de Cayatte de la narration vidéoludique par l'ajout des événements d'apprentissages-enseignements (tableau $n^{\circ} 2$ ), nous énonçons qu'un discours est le produit de controverses entre:

- l'expérience-cadre qui mobilise dans différentes mesures des éléments de discours et des actions vidéoludiques (code et process selon Galloway) ancrés dans des approches pédagogiques (transmission, documentation, etc.)

- l'opérateur (les procédures) qui par ses actions (play et algorithm) applique ou non les comportements attendus (réception, exploration, etc.) ${ }^{26}$

- durant une succession de situations-séquences. L'ensemble aboutit alors à des moments de jeu permettant ou non la révélation d'un discours contenu dans un jeu (figure $n^{\circ} 3$ ). ${ }^{26}$ Ils et elles peuvent aussi notamment détourner l'expérience, ne pas respecter les consignes ou
tout simplement refuser de jouer. 


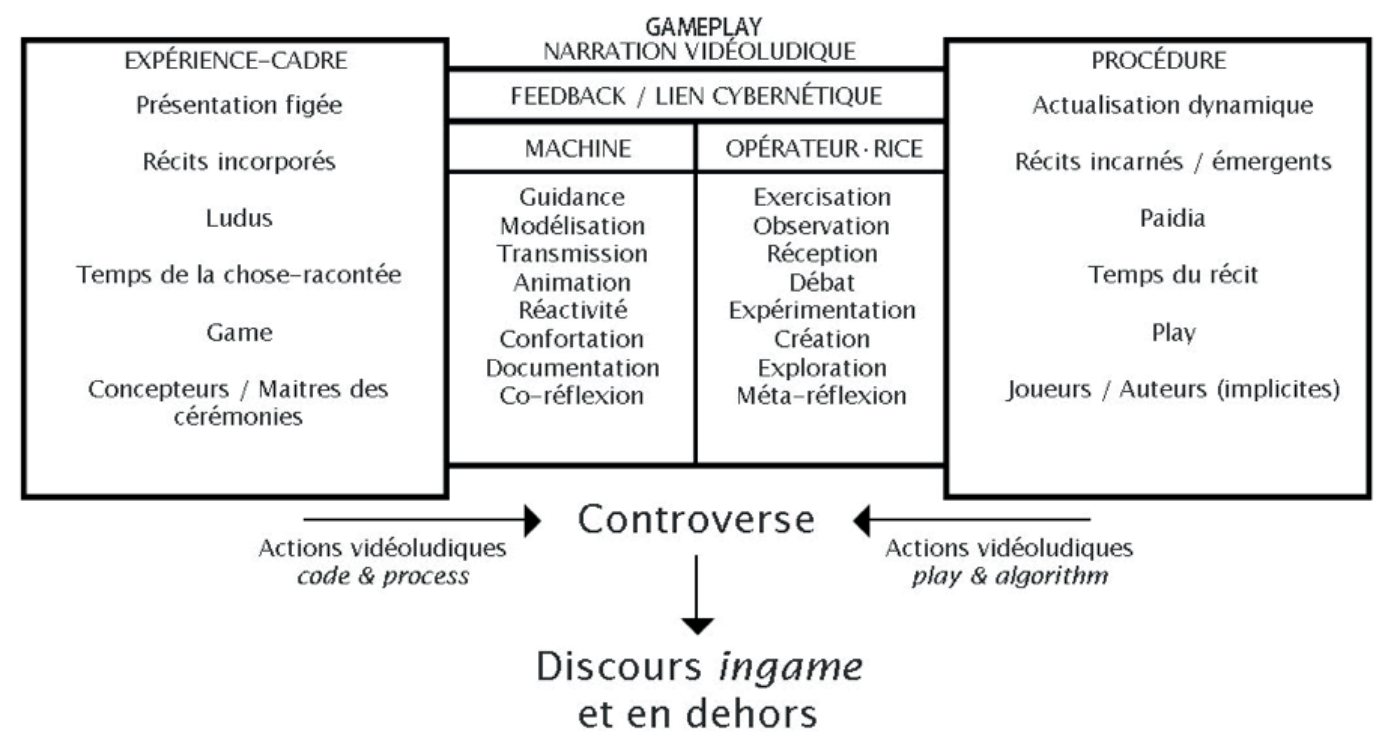

FIGURE N ${ }^{\circ} 3: L^{2}$ 'ÉMERGENCE DES DISCOURS À PARTIR DES CONTROVERSES ENTRE L'EXPÉRIENCE-CADRE ET LES PROCÉDURES

Un des points à développer concerne la non-prise en compte de la multitude de profils de joueurs. À ce jour, la littérature à propos des typologies de joueurs et joueuses est foisonnante, en témoigne la méta-synthèse de Janne Tuunanen et Juho Hamari (Tuunanen et Hamari, 2012). Cependant, il apparait que la majeure partie des typologies porte sur la catégorisation d'attitudes (comportementales et psychologiques). C'est pourquoi nous proposons plutôt d'ouvrir une réflexion sur la formalisation d'un outil permettant d'évaluer les compétences - des savoir-agir complexes mobilisant et combinant des ressources internes et externes (Tardif, 2006) - des joueurs. Cet outil pourrait prendre la forme d'un référentiel de compétences ${ }^{27} \mathrm{du}$ joueur-modèle. Il serait intéressant alors de croiser la persuasivité d'un discours avec le degré de compétences d'un joueur ou d'une joueuse ${ }^{28}$ afin d'offrir une nouvelle perspective socio-pragmatique sur l'étude des discours vidéoludiques.

\footnotetext{
27 Un exemple de compétences, selon le formalisme de Jacques Tardif (Tardif, 2006), serait par exemple: « analyser les discours contenus dans un jeu ». L'enjeu du référentiel serait alors de formaliser des étapes formant une trajectoire de développement mais aussi d'énoncer les situations pragmatiques dans lesquelles le joueur mobiliserait cette compétence.

28 Par ailleurs, il sera dans ce cadre important de proposer des modalités d'évaluation des compétences. Cela peut passer par des tests d'auto-positionnement ou la mise en place d'un portfolio amenant alors des preuves constatables d'une compétence.
} 


\section{Bibliographie}

Amossy R. (2014), « L'éthos et ses doubles contemporains. Perspectives disciplinaires ». Langage et société, $\mathrm{n}^{\circ} 149$, p. 13-30.

Aufreiter N. et al. (2012), Seven Strategies to Beat the Retail Apocalypse. McKinsey \& Company. En ligne, consulté le 8 juin 2019. URL : https://www.mckinsey.com/ / media/mckinsey/dotcom/client_service/Marketing\%20and\%20Sales/PDFs/ McKinsey\%20CMSO\%20forum\%20-\%20Seven\%20strategies\%20to\%20beat\%20 the\%20retail\%20apocalypse.ashx.

Auray N., Vetel B. (2013), « L'exploration comme modalité d'ouverture attentionnelle. Design et régulation d'un jeu freemium », Réseaux, vol. 182, nº 6, p.153-186.

Barnabé F., Delbouille J. (2018), « Aux frontières de la fiction : l'avatar comme opérateur de réflexivité », Sciences du jeu, n ${ }^{\circ}$. En Ligne, consulté le 8 juin 2018. URL : https:// journals.openedition.org/sdj/958.

Beal V., Fol S., Rousseau M. (2016), " De quoi le "smart shrinkage” est-il le nom ? Les ambiguïtés des politiques de décroissance planifiée dans les villes américaines », Géographie, économie, société, vol. 18, nº 2, p. 211-234.

Bogost I. (2007), Persuasive Games: The Expressive Power of Videogames, Cambridge, MA, The MIT Press.

Bricongne J.-C., Lapegue V., Monso O. (2009), " La crise des "subprimes" : de la crise financière à la crise économique ", note de conjoncture. Paris, INSEE. En ligne, consulté le 23 février 2019. URL : https://www.insee.fr/fr/ statistiques/1407817?sommaire=1407821.

CAYATte R. (2018), « Temps de la chose-racontée et temps du récit vidéoludique : comment le jeu vidéo raconte ? ", Sciences $d u$ jeu, $n^{\circ}$ 9. En Ligne, consulté le 8 juin 2018. URL : https://journals.openedition.org/sdj/936.

Filippo L. D., Schmoll P. (2016), « La ville après l'apocalypse. Entre formalisation projective et réalisation locale », Revue des Sciences sociales, n 56, p. 126-133.

Galloway A. R. (2006), Gaming : Essays on Algorithmic Culture, Minneapolis, University of Minnesota Press.

Genvo S. (2013), Penser la formation et les évolutions du jeu sur support numérique, Mémoire pour l'habilitation à diriger des recherches en sciences de l'information et de la communication, Metz, Université de Lorraine.

Genvo S. (2016), « Defining and Designing Expressive Games: the Case of Keys of a Gamespace », Kinephanos, numéro spécial, avril 2016.

GiNER, E. (2017), « Inciter à la réflexivité par les mécanismes ludiques : une analyse comparée de The Witness, Undertale et The Beginner's Guide », Le Pardaillan, $n^{\circ} 2$. p.167-181. En ligne, consulté le 8 juin 2019. URL : https://halshs.archives-ouvertes.fr/ halshs-01622006/document/. 
GrandjeAn G. (2019), "Ordre et désordre de l'espace social vidéoludique, analyse formelle et visuelle de quelques villages de The Legend of Zelda : Breath of the Wild», Émulations, $\mathrm{n}^{\circ} 30$.

Henriot J. (1989), Sous couleur de jouer : La métaphore ludique, Paris, José Corti Éditions.

Houssaye J. (dir.) (1993), Pédagogie : une encyclopédie pour aujourd'hui, Montrouge, ESF Éditeur.

Hudson L. (2017), « Coming to Video Games Near You: Depressed Towns, Dead-End Characters », The New York Times. En ligne, consulté le 23 février 2019. URL : https:// www.nytimes.com/2017/02/15/technology/personaltech/coming-to-video-gamesnear-you-depressed-towns-dead-end-characters.html/.

LAurent S. (2018), « Le peuple de Trump », Le Débat, vol. 198, n 1, p. 4-10.

Leclerce D., Poumay M. (2008), « Le Modèle des Événements d’Apprentissage Enseignement ». En ligne, consulté le 23 février 2019. URL : https://orbi.uliege.be/ handle/2268/13968.

Rusch D. (2009), "Mechanisms of the Soul : Tackling the Human Condition in Videogames », DiGRA '09 - Proceedings of the 2009 DiGRA International Conference: Breaking New Ground: Innovation in Games, Play, Practice and Theory, vol. 5, Londres, Université Brunel.

SoHier R. (2016), «L’expérience émersive du jeu vidéo », Implications philosophiques. En ligne, consulté le 24 février 2019. URL : http://www.implications-philosophiques. org/actualite/une/lexperience-emersive-du-jeu-video/.

TARDIF J. (2006), L'évaluation des compétences : Documenter le parcours de développement, Montréal, Chenelière Education.

Trepanier-Jobin G. (2016), « Differentiating Serious, Persuasive, and Expressive Games », Kinephanos, numéro spécial, avril 2016.

Tuunanen J., Hamari J. (2012), " Meta-synthesis of player typologies », DiGRA Nordic '12: Proceedings of 2012 International DiGRA Nordic Conference, vol. 10. Tampare, Université de Tampere, Université de Jyväskylä et Université de Turku. 


\section{Annexes}

Annexe 1 : Le diagramme de LUdicisation, GenVo, 2013

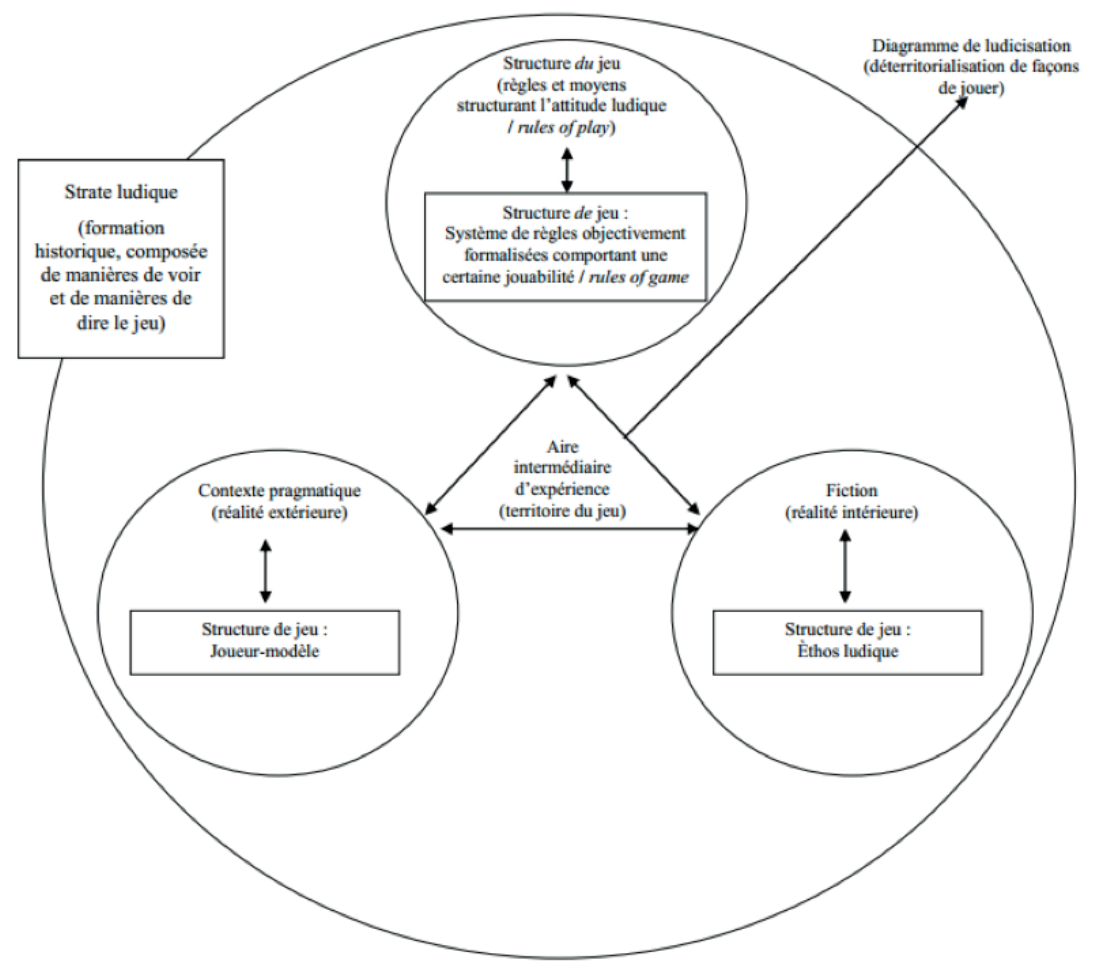

Annexe 2 : Gameplay et narRation vidéoludique, Cayatte, 2018

\section{Expérience-Cadre}

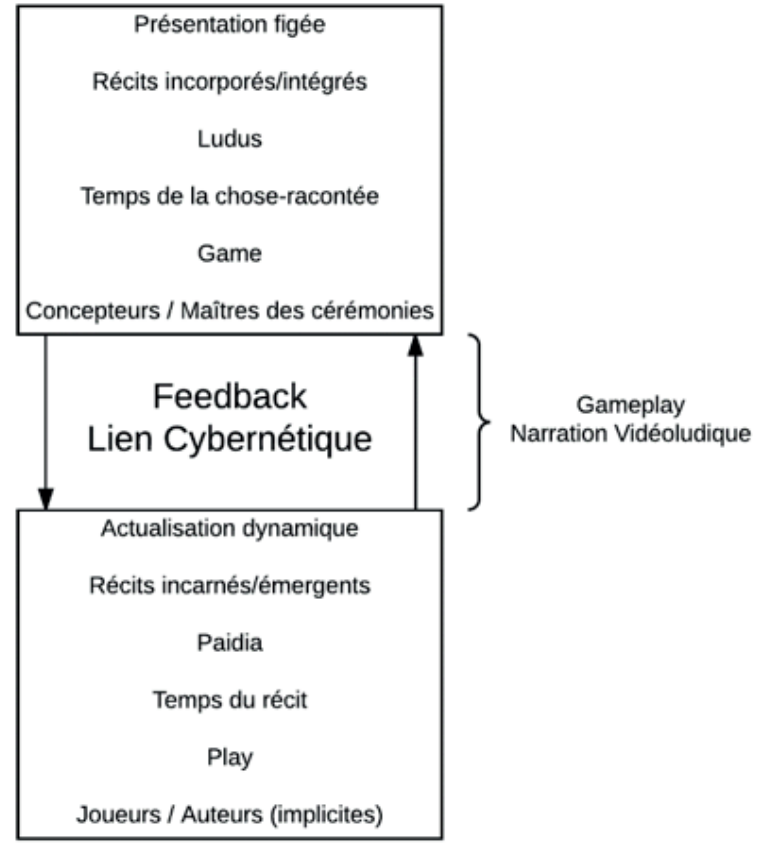

Procédure 